\title{
Effect of Sputtering Power on Optical and Electrical Properties of Indium Tin Oxide Films
}

\author{
Shiuh-Chuan Her* and Chun-Fu Chang \\ Department of Mechanical Engineering, Yuan Ze University, Chung-Li 320, Taiwan
}

(Received December 21, 2015; accepted May 25, 2016)

Keywords: indium tin oxide, transmittance, sputtering power, resistivity

Indium tin oxide (ITO) films were deposited onto a glass substrate by direct current (DC) magnetron sputtering. The effect of the sputtering power on the microstructure, electrical, and optical electrical properties was examined. Experimental results indicated that increasing the sputtering power caused surface roughness to decrease while the resistivity increased. The average of the optical transmittance in the wavelength region of 300-1200 nm for ITO films decreased from 77 to $73 \%$ as the sputtering power increased from 50 to $200 \mathrm{~W}$. Results of this work suggested that a better performance of both optical transparency and electrical conductivity of ITO films can be achieved by operating at a low level of sputtering power.

\section{Introduction}

Transparent conducting oxide (TCO) films with high optical transmittance and electrical conductivity in the visible wavelength region play an important role in many engineering applications. ${ }^{(1)}$ Indium tin oxide (ITO), with high optical transparency and electrical conductivity, is one of the most widely used TCO materials. It has been employed as a transparent conducting layer in a variety of photoelectronic components and devices such as solar cells, ${ }^{(2)}$ organic light emitting diodes, ${ }^{(3)}$ gas sensors, ${ }^{(4)}$ and liquid crystal displays. ${ }^{(5)}$ There are many manufacturing techniques that have been proposed to fabricate ITO films, such as pulsed laser deposition, ${ }^{(6)}$ electron beam deposition, ${ }^{(7)}$ sputtering,${ }^{(8)}$ and the sol-gel method. ${ }^{(9)}$ Among the techniques available for preparing ITO films, sputtering, with good reliability, high growth rate, and better control of the film characteristics, is one of the most often used methods. ${ }^{(10)}$ Guillen and Herrero ${ }^{(11)}$ investigated the effect of the substrate on the structure, morphology, electrical, and optical characteristics of ITO films prepared by sputtering at room temperature on glass and polymer substrates. Their results showed that ITO films deposited onto a polymer exhibited larger grain size and higher conductivity then those on a glass substrate. Jafan et al. ${ }^{(12)}$ studied the influence of thermal decomposition temperature on the microstructure, optical, electrical, and morphological characteristics of ITO thin films prepared by a sol-gel spin coating process. They found that ITO films pyrolyzed at $500{ }^{\circ} \mathrm{C}$ had the best electrical conductivity and crystal structure. Giusti et al. ${ }^{(13)}$ evaluated the influences of substrate temperature and oxygen pressure on microstructures, morphologies, optical, and electrical properties of ITO films deposited by a pulsed laser system. The best performance in terms of

"Corresponding author: e-mail: mesch@saturn.yzu.edu.tw 
high optical transmittance and low electrical resistivity was achieved for ITO films prepared at a deposition temperature of $200^{\circ} \mathrm{C}$ with oxygen pressure ranging from 0.66 to $2 \mathrm{~Pa}$.

In this work, the influence of sputtering power on the surface morphology, optical transmittance, and electrical resistivity of ITO films was studied. ITO films were grown on glass substrates by magnetron sputtering operating at direct current. The surface roughness and microstructure morphology of ITO films were evaluated using atomic force microscopy (AFM). Optical transmittance was measured by a Lambda 750 spectrometer from Perkin Elmer. Electrical properties were investigated using the Hall measurement.

\section{Experimetal Methods}

A series of ITO films were prepared on glass substrates using DC magnetron sputtering (ULVAC MB06-4703) at different sputtering powers. The target ITO was a disk $50.8 \mathrm{~mm}$ in diameter and $3 \mathrm{~mm}$ thick with a composition of $\mathrm{In}_{2} \mathrm{O}_{3} 90 \mathrm{wt} \%$ and $\mathrm{SnO}_{2} 10 \mathrm{wt} \%$. The substrate and target were kept at a distance of $10 \mathrm{~cm}$. Before deposition, the substrate was cleaned with a soap solution and rinsed with distilled water. The substrate was ultrasonically cleaned in acetone and rinsed in an ultrasonic bath for $15 \mathrm{~min}$. After that, the substrate was placed in an oven to dry at a temperature of $50{ }^{\circ} \mathrm{C}$ for $30 \mathrm{~min}$ prior to the deposition process. To obtain the required vacuum, a combination of rotary and turbo pumps were used to evacuate the chamber. The pressure of the chamber was reduced to $8 \times 10^{-4} \mathrm{~Pa}$ after pumping for $90 \mathrm{~min}$. Highly pure argon $(99.999 \%)$ was introduced into the chamber at a flow rate of $10 \mathrm{sccm}$ and acted as the sputtering gas. Prior to deposition, the substrate and target were pre-sputtered with an Ar plasma for 20 min to clean the surfaces. During the period of the deposition process, the operating pressure and deposition temperature were kept at $2 \times 10^{-1} \mathrm{~Pa}$ and $25^{\circ} \mathrm{C}$, respectively, for all samples. Following the same process, four different ITO films were deposited onto glass substrates with four different sputtering powers of 50, 100, 150 , and $200 \mathrm{~W}$. The effect of sputtering power on the microstructure, optical transmittance, and electrical resistivity of the ITO film was studied. It is well known that the thickness of the film depends on the sputtering power. A higher sputtering power leads to a higher kinetic energy of the ad-atoms deposited onto the glass substrate resulting in an increase in the film thickness. In this work, the thicknesses for all the testing samples were $200 \mathrm{~nm}$. To maintain this same thickness, the sputtering durations for sputtering powers of 50,100, 150, and $200 \mathrm{~W}$ were 48, 24, 15, and 12 min, respectively. A surface profiler (KLA Tencor P16) was used to measure the thickness of the ITO films. Before the deposition process, a tiny tape was attached to each of the four corners of the square substrate to create a small step on the surface of the film as shown in Fig. 1. The height of the step at the four corners of the substrate was measured and the thickness of the film reported in this paper was the average of these values. The thicknesses of ITO films deposited with sputtering powers of $50,100,150$, and $200 \mathrm{~W}$ are 202, 203, 198, and $199 \mathrm{~nm}$, respectively.

\section{Results and Discussions}

\subsection{Surface topography}

The surface topography and microstructure of ITO films were evaluated using atomic force microscopy (Seiko Instruments Inc. SPA 400). Figure 2 shows the surface morphology of ITO films deposited at various sputtering powers. The roughness (reported as the root mean square) of 


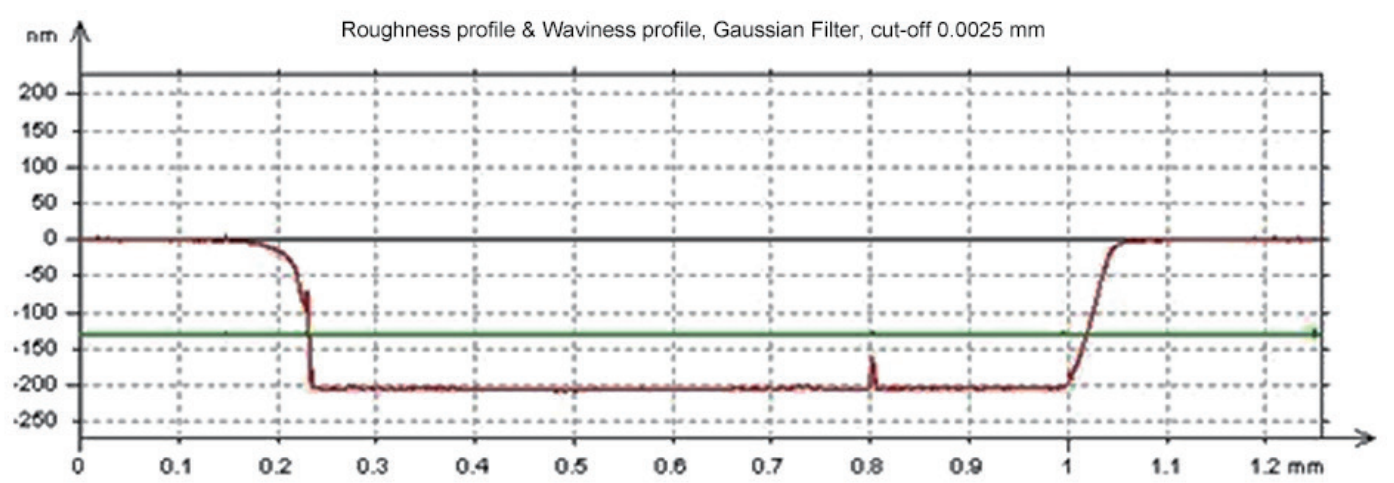

Fig. 1. (Color online) Surface profile of an ITO film.

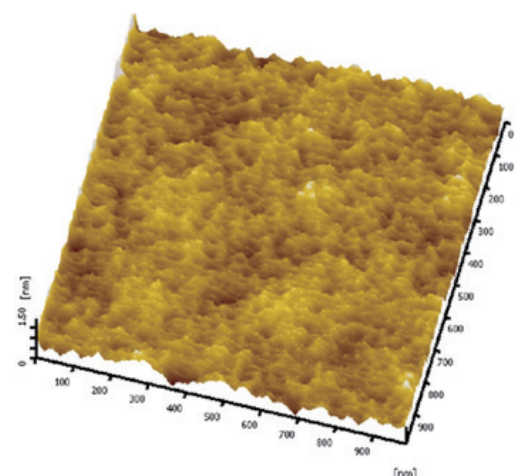

(a)

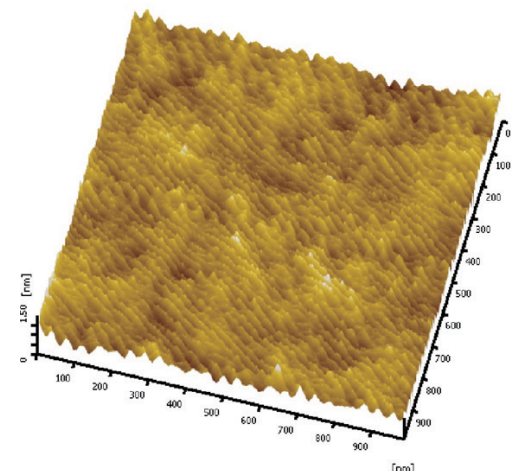

(c)

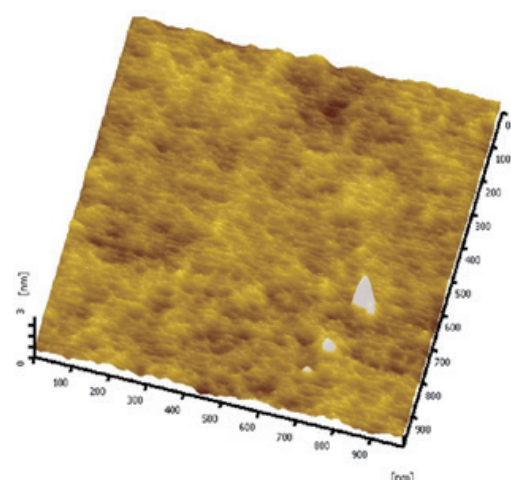

(b)

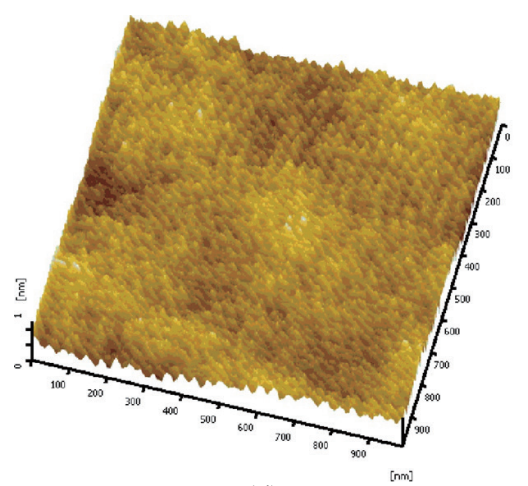

(d)

Fig. 2. (Color online) Surface topography of ITO films deposited at different sputtering powers. (a) 50, (b) 100, (c) 150 , and (d) $200 \mathrm{~W}$.

ITO films prepared at sputtering powers of $50,100,150$, and $200 \mathrm{~W}$ was $0.353,0.242,0.204$, and $0.152 \mathrm{~nm}$, respectively. The results indicate that increasing the sputtering power causes a decrease in the surface roughness of ITO films. The morphology of ITO films as shown in Fig. 2 exhibited uniformly distributed collar grains with fine crystallinity. The figure shows that a highly compact and dense film can be achieved. As the sputtering power is increased, more kinetic energy is introduced into the deposited atoms, leading to an increase in the migration mobility, which results 
in a decrease of surface roughness. Castro and Tavares ${ }^{(14)}$ also reported that, at higher substrate temperature, the sputtered particles have more energy to diffuse, resulting in the formation of a more compact film.

\subsection{Optical properties}

The optical properties of the films can be examined using spectroscopic ellipsometry. In this study, the optical transmittance and reflectance spectra were measured by a double beam Lambda 750 spectrometer from Perkin Elmer. Figure 3 shows the optical transmittance spectrum in the wavelength region of $300-1200 \mathrm{~nm}$ for ITO films prepared at various sputtering powers. The transmittance spectrum in the range of 300-400 nm is plotted in Fig. 4. It shows a blue shift of the transmittance spectrum as the sputtering power is decreased. The average transmittances of ITO films deposited at different sputtering powers are presented in Table 1. The data show that the average optical transmittance of ITO films decreased from 77 to $73 \%$ in the wavelength region of $300-1200 \mathrm{~nm}$ and decreased from 81 to $77 \%$ in the visible wavelength region of $400-800 \mathrm{~nm}$, as the sputtering power was increased from 50 to $200 \mathrm{~W}$. Similar results were found by Lee et al. . $^{(15)}$ The reflectance spectra of ITO films are plotted in Fig. 5. Table 2 lists the average reflectance of ITO films. The data show that the average reflectance of ITO films increased from 15.8 to $17.92 \%$ in the wavelength region of 300-1200 nm and increased from 18.32 to $19.35 \%$ in the visible wavelength region of $400-800 \mathrm{~nm}$ as the sputtering power was increased from 50 to $200 \mathrm{~W}$.

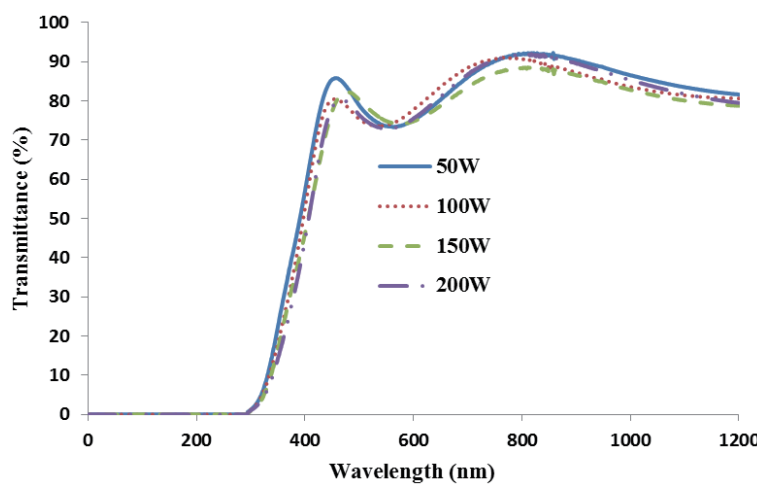

Fig. 3. (Color online) Optical transmittance spectra of ITO films prepared at various sputtering powers.

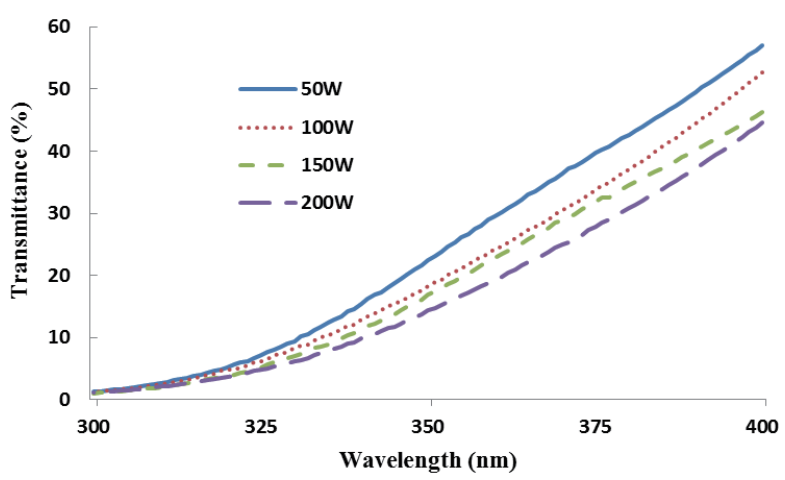

Fig. 4. (Color online) Optical transmittance spectra in the wavelength range of $300-400 \mathrm{~nm}$ for ITO films prepared at various sputtering powers.

Table 1

Average optical transmittance of ITO films prepared at various sputtering powers.

\begin{tabular}{lcccc}
\hline \multirow{2}{*}{$\begin{array}{l}\text { Wavelength } \\
\text { range }\end{array}$} & $50 \mathrm{~W}$ & $100 \mathrm{~W}$ & $150 \mathrm{~W}$ & $200 \mathrm{~W}$ \\
\cline { 2 - 5 } $300-1200 \mathrm{~nm}$ & $77.20 \%$ & $75.662 \%$ & $73.88 \%$ & $73.05 \%$ \\
$400-800 \mathrm{~nm}$ & $80.88 \%$ & $80.66 \%$ & $78.25 \%$ & $77.06 \%$ \\
\hline
\end{tabular}




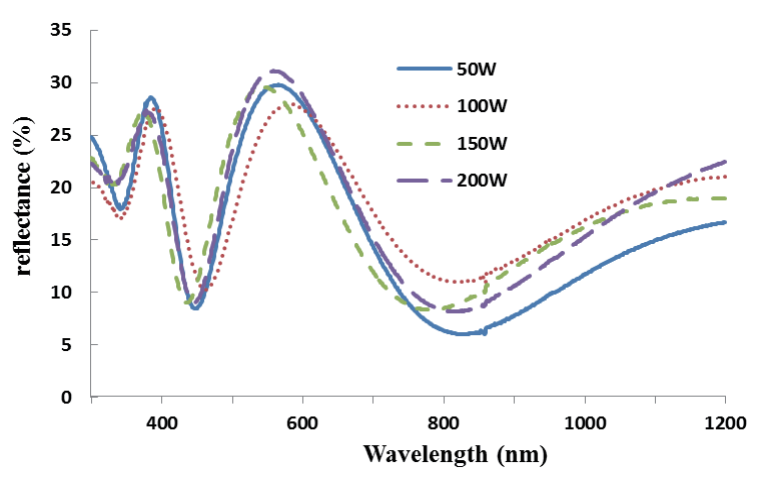

Fig. 5. (Color online) Optical reflectance spectra of ITO films prepared at various sputtering powers.

Table 2

Average optical reflectance of ITO films prepared at various sputtering powers.

\begin{tabular}{lcccc}
\hline Wavelength & \multicolumn{4}{c}{ Sputtering power } \\
\cline { 2 - 5 } range & $50 \mathrm{~W}$ & $100 \mathrm{~W}$ & $150 \mathrm{~W}$ & $200 \mathrm{~W}$ \\
\hline $300-1200 \mathrm{~nm}$ & $15.80 \%$ & $18.25 \%$ & $17.24 \%$ & $17.92 \%$ \\
$400-800 \mathrm{~nm}$ & $18.32 \%$ & $19.32 \%$ & $17.73 \%$ & $19.35 \%$ \\
\hline
\end{tabular}

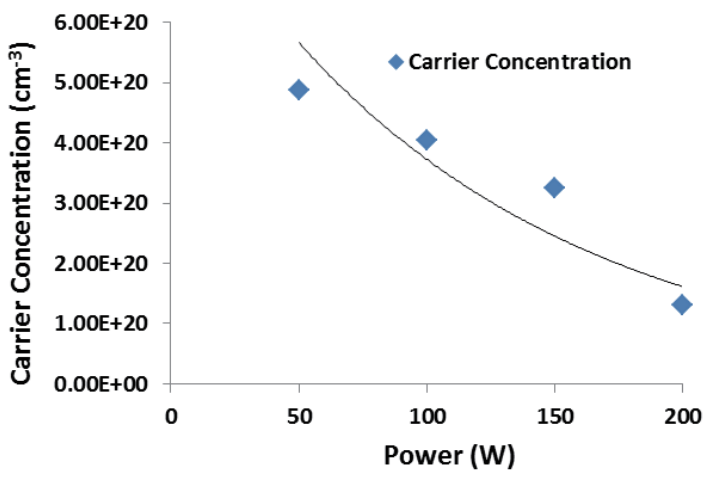

Fig. 6. (Color online) Variation in carrier concentration of ITO film as a function of sputtering power.

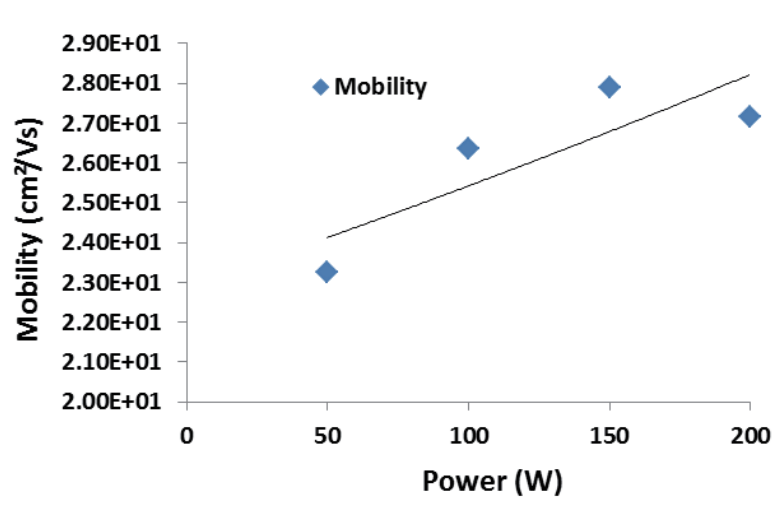

Fig. 7. (Color online) Variation in mobility of ITO film as a function of sputtering power.

\subsection{Electrical properties}

The resistivity of ITO films was evaluated using Hall effect measurements at room temperature to characterize the electrical properties using an Ecopia HMS-300 system. The measurements of electrical resistivity for each sample were repeated several times. The resistivities of ITO films deposited at sputtering powers of 50,100,150, and $200 \mathrm{~W}$ are $5.702 \times 10^{-4}, 5.798 \times 10^{-4}, 7.011 \times$ $10^{-4}$, and $2.376 \times 10^{-4} \Omega$-cm, respectively. The carrier concentration decreased from $4.88 \times 10^{20}$ to 1.30 $\times 10^{20} \mathrm{~cm}^{-3}$ as the sputtering power increased from 50 to $200 \mathrm{~W}$ as shown in Fig. 6 . The mobility of ITO films as a function of sputtering power is plotted in Fig. 7. The electrical properties including resistivity, carrier concentration, and mobility of ITO films prepared at sputtering powers of 50 , 
Table 3

Electrical properties of ITO films prepared at various sputtering powers.

\begin{tabular}{lcccc}
\hline \multirow{2}{*}{ Electrical properties } & $50 \mathrm{~W}$ & $100 \mathrm{~W}$ & $150 \mathrm{~W}$ & $200 \mathrm{~W}$ \\
\cline { 2 - 5 } & 5.702 & 5.798 & 7.011 & 23.76 \\
Resistivity $\left(10^{-4} \Omega \cdot \mathrm{cm}\right)$ & 4.880 & 4.047 & 3.254 & 1.299 \\
Carrier concentration $\left(10^{20} \mathrm{~cm}^{-3}\right)$ & 23.25 & 26.35 & 27.88 & 27.16 \\
Mobility $\left(\mathrm{cm}^{2} / \mathrm{Vs}\right)$ & & & & \\
\hline
\end{tabular}

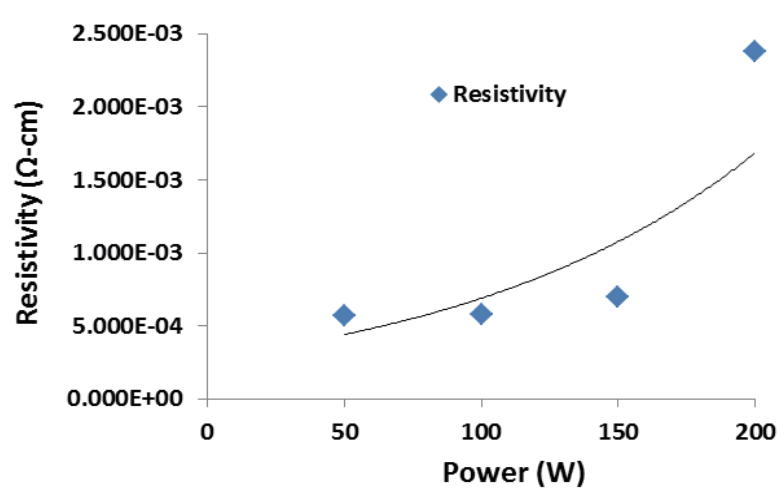

Fig. 8. (Color online) Variation in resistivity of ITO film as a function of sputtering power.

100,150 , and $200 \mathrm{~W}$ are listed in Table 3 . The decrease in the resistivity as the sputtering power was decreased is shown in Fig. 8 and can be considered the result of the increase in the crystallinity. Lee et al. ${ }^{(15)}$ also found that the resistivity of ITO film decreased with a decrease in the sputtering power. Sujatha et al. ${ }^{(16)}$ reported that the crystallinity of ITO films can be improved with substrate bias. Ion bombardment on the substrates due to the sputtering power has effects similar to that of substrate bias.

\section{Conclusions}

ITO films were grown on glass substrates by direct current magnetron sputtering at a variety of sputtering powers ranging from 50 to $200 \mathrm{~W}$. The effect of the sputtering power on the surface morphology, electrical, and optical properties was studied. AFM measurements showed that the surface roughness decreased as the sputtering power was increased. Optical transmittance decreased as the sputtering power was increased. Experimental results show that optical transmittance averaging $80 \%$ in the visible region was achieved for ITO films prepared at a sputtering power of $50 \mathrm{~W}$. The electrical resistivity increased as the sputtering power was increased. A decrease in the sputtering power enhances the performance of ITO films by producing a lower resistivity and a higher optical transmittance.

\section{Acknowledgements}

The authors thank the Ministry of Science and Technology of the R.O.C. for financial support under grant no. MOST 104-2221-E155-057-MY3. 


\section{References}

1 I. Maksimenko, M. Gross, T. Königer, H. Münstedt, and P. J. Wellmann: Thin Solid Films 518 (2010) 2910.

2 L. Schmidt-Mende, A. Fechtenkotter, K. Mullen, E. Moons, R. H. Friend, and J. D. Mackenzre: Physica E 14 (2002) 263.

3 H. P. Tang, H. X. Liao, K. Xu, Z. X. Zhou, and L. H. Zhu: J. Lumin. 118 (2006) 39.

4 Z. Jiao, M. H. Wu, J. Z. Gu, and X. L. Sun: Sens. Actuators, B 94 (2003) 216.

5 Z. H. Li, Y. P. Ke, and D. Y. Ren: Trans. Nonferrous Met. Soc. China 18 (2008) 366.

6 S. H. Kim, N. M. Park, T. Y. Kim, and G. Y. Sung: Thin Solid Films 475 (2005) 262.

7 H. R. Fallah, M. Ghaseni, A. Hassanzadeh, and H. Steki: Physica B 373 (2006) 274.

8 S. Boycheva, A. K. Sytchkova, M. L. Grilli, and A. Piegari: Thin Solid Films 515 (2007) 8469.

9 J. Liu, D. Wu, and S. Zeng: J. Mater. Process. Technol. 209 (2009) 3943.

10 F. Zhua, C. H. A. Huan, K. Zhang, and A. T. S. Wee: Thin Solid Films 359 (2000) 244.

11 C. Guillen and J. Herrero: Thin Solid Films 480-481 (2005) 129.

12 M. M. H. Jafan, M. R. Zamani-Meymian, R. Rahimi, and M. Rabbani: Microelectron. Eng. 130 (2014) 40.

13 G. Giusti, L. Tian, I. P. Jones, J. S. Abell, and J. Bowen: Thin Solid Films 518 (2009) 1140.

14 M. V. Castro and C. J. Tavares: Thin Solid Films 586 (2015) 13.

15 J. Lee, H. Jung, J. Lee, D. Lim, K. Yang, J. Yi, and W. C. Song: Thin Solid Films 516 (2008) 1634.

16 Ch. Sujatha, G. Mohan Rao, and S. Uthanna: Mater. Sci. Eng., B 94 (2002) 106. 